\title{
Improved Bit-Flipping Algorithm for Successive Cancellation Decoding of Polar Codes
}

\author{
Furkan Ercan, Student Member, IEEE, Carlo Condo, Member, IEEE, and Warren J. Gross Senior Member, IEEE
}

\begin{abstract}
The interest in polar codes has been increasing significantly since their adoption for use in the $5^{\text {th }}$ generation wireless systems standard. Successive cancellation (SC) decoding algorithm has low implementation complexity, but yields mediocre error-correction performance at the code lengths of interest. SC-Flip algorithm improves the error-correction performance of SC by identifying possibly erroneous decisions made by $\mathrm{SC}$ and re-iterates after flipping one bit. It was recently shown that only a portion of bit-channels are most likely to be in error. In this work, we investigate the average log-likelihood ratio (LLR) values and their distribution related to the erroneous bitchannels, and develop the Thresholded SC-Flip (TSCF) decoding algorithm. We also replace the LLR selection and sorting of SCFlip with a comparator to reduce the implementation complexity. Simulation results demonstrate that for practical code lengths and a wide range of rates, TSCF shows negligible loss compared to the error-correction performance obtained when all singleerrors are corrected. At matching maximum iterations, TSCF has an error-correction performance gain of up to $0.45 \mathbf{d B}$ compared with SC-Flip decoding. At matching error-correction performance, the computational complexity of TSCF is reduced by up to $40 \%$ on average, and requires up to $5 \times$ lower maximum number of iterations.
\end{abstract}

Index Terms-polar codes, SC-Flip decoding, 5G, errorcorrection performance.

\section{INTRODUCTION}

$\mathbf{P}$ OLAR codes, introduced by Arikan in [1], are a class of linear block codes that provably achieve channel capacity. Due to their low-complexity encoding and decoding, polar codes have been selected as a coding scheme in the $5^{\text {th }}$ generation wireless systems standards $(5 \mathrm{G})$. Current use of polar codes within $5 \mathrm{G}$ includes the enhanced mobile broadband (eMBB) control channel [2], while they are being considered for ultra-reliable low-latency communications (URLLC) and massive machine-type communications (mMTC). 5G communications require decoding algorithms that have improved error-correction performance and throughput, along with reduced power and energy consumption compared to previous communication standards.

Successive-cancellation (SC) decoding is the first decoding algorithm for polar codes, proposed in [1]. SC decoding is able to achieve channel capacity as the codeword length tends to infinity; however, its error-correction performance is degraded at practical lengths. To tackle this issue, SC-List decoding algorithm was proposed in [3]. SC-List decoders improve the

F. Ercan and W. J. Gross are with the Department of Electrical and Computer Engineering, McGill University, Montréal, Québec, Canada. e-mail: furkan.ercan@mail.mcgill.ca, warren.gross@mcgill.ca. C. Condo was with the Department of Electrical and Computer Engineering, McGill University. Now he is with the Huawei Paris Research Center, Boulogne-Billancourt, France. e-mail: carlo.condo@huawei.com
SC error-correction performance at the cost of longer latency, as well as increased area occupation and power consumption. Several contributions have improved latency, area occupation and power consumption of SC-List algorithm [4]-[8].

The SC-Flip decoding algorithm takes an alternative approach to the improvement of SC [9]. In contrast to the parallel SC decoding approach of SC-List, SC-Flip relies on multiple subsequent decoding attempts in order to identify and correct the wrong decision made by SC, sequentially flipping unreliable bits. It yields an average computational complexity equivalent to that of SC decoding at medium to high signal-tonoise ratio (SNR) values, mainly sacrificing worst case latency. Its error-correction performance is comparable to that of SCList decoding with a list size of 2 at practical iteration lengths. Modifications to SC-Flip decoding that were carried out in [10]-[14] have shown that the error-correction performance can be greatly improved.

In [12], two methods were proposed to improve SC-Flip decoding, based on the distribution of first wrong bit estimation, that is always caused by channel noise. One method simplifies the operations required by SC-Flip decoding, without degrading the error-correction performance. The second method restricts the search space for bit-flipping, improving the error-correction performance. These techniques are very effective at low code rates, but their gain degrades quickly as the code rate increases.

The focus of this work is to identify and correct the first channel-induced error as efficiently as possible. Our approach is based on a critical set of bit indices that are more errorsusceptible than the rest, similar to [12]. We summarize the contributions of this work as follows:

- The log-likelihood ratio (LLR) values of the critical set are observed, noting that the mean values of LLRs of critical set for successful and unsuccessful decoding differ substantially.

- Using this information on a critical set, an LLR threshold is applied to further restrict the search space for bitflipping; with this technique, SC-Flip is able to approach the error-correction performance obtained when all first channel-induced errors are corrected, with a small number of iterations at high, medium and low code rates. We name this new decoding approach as Thresholded SCFlip (TSCF) algorithm. Simulations show that TSCF has negligible loss compared to the error-correction performance that can be achieved when all single-channelinduced errors are corrected.

- In TSCF decoding, we replace the the LLR selection and sorting of the original SC-Flip with a simple threshold 


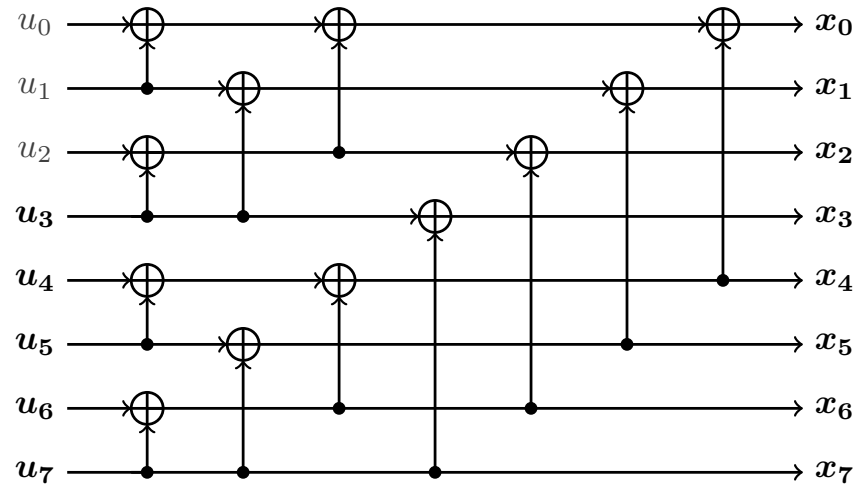

Fig. 1: Polar code encoding for $P C(8,5)$.

comparator to further reduce the implementation complexity.

- We show that although the observation of the critical set depends on the simulated $E_{b} / N_{0}$, a single critical set valid for all SNRs is sufficient to substantially improve the error-correction performance compared to the baseline SC-Flip decoding.

The rest of this work is organized as follows: In Section II, encoding and decoding techniques of polar codes are reviewed. In Section III, the proposed SC-Flip with thresholded index selection is described. Simulation results are presented in Section IV, and conclusions are drawn in Section V.

\section{PRELIMINARIES}

\section{A. Polar Codes}

A polar code $P C(N, K)$ of code length $N$ and rate $R=$ $K / N$ is a linear block code that divides $N=2^{n}, n \in \mathbb{Z}^{+}$ bit-channels in $K$ reliable ones and $N-K$ unreliable ones. Information bits are transmitted via the reliable channels. The unreliable channels are fixed to a value which is known by both the transmitter and the receiver, usually zero. They are thus called frozen channels.

Polar codes are encoded through the following matrix multiplication:

$$
x_{0}^{N-1}=u_{0}^{N-1} G^{\otimes n},
$$

where $\boldsymbol{x}_{\mathbf{0}}^{\boldsymbol{N}-\mathbf{1}}=\left\{x_{0}, x_{1}, \ldots, x_{N-1}\right\}$ represents the encoded vector, $\boldsymbol{u}_{\mathbf{0}}^{N-1}=\left\{u_{0}, u_{1}, \ldots, u_{N-1}\right\}$ is the input vector, and the generator matrix $G^{\otimes n}$ is obtained as the $n$-th Kronecker product of the polarization matrix $G=\left[\begin{array}{ll}1 & 0 \\ 1 & 1\end{array}\right]$. Due to the recursive nature of the encoding process, an $N$-length polar code can be interpreted as the concatenation of two polar codes of length $N / 2$. The encoding operation in (1) for polar code $P C(8,5)$ is portrayed in Fig. 1; gray indices represent the frozen bits whereas the black indices indicate the information bits.

The scheduling of operations required by the SC decoding algorithm allows to see its process as a binary tree search, where the tree is explored depth-first, with priority given to the left branch. Fig. 2 portrays an example of SC decoding tree, for $P C(8,5)$. Each node receives from its parent a vector

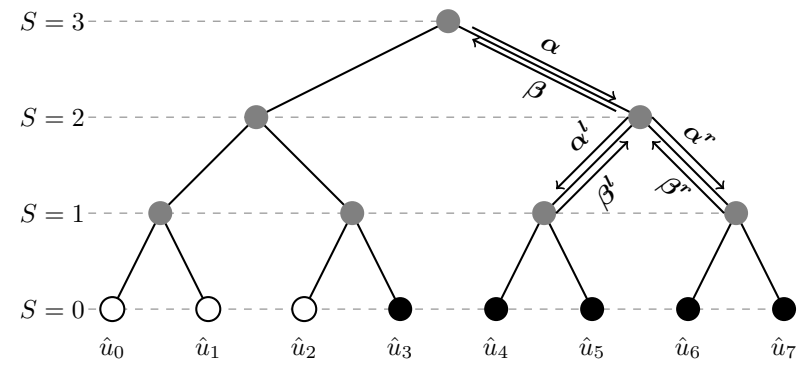

Fig. 2: Successive-cancellation decoding tree for a $P C(8,5)$ code.

of LLRs $\boldsymbol{\alpha}=\left\{\alpha_{0}, \alpha_{1}, \ldots, \alpha_{2^{S}-1}\right\}$. Each node at stage $S$ computes the left $\boldsymbol{\alpha}^{\boldsymbol{l}}=\left\{\alpha_{0}^{l}, \alpha_{1}^{l}, \ldots, \alpha_{2^{S-1}-1}^{l}\right\}$ and right $\boldsymbol{\alpha}^{\boldsymbol{r}}=$ $\left\{\alpha_{0}^{r}, \alpha_{1}^{r}, \ldots, \alpha_{2^{S-1}-1}^{r}\right\}$ LLR vectors sent to child nodes as

$$
\begin{aligned}
& \alpha_{i}^{l}=\operatorname{sgn}\left(\alpha_{i}\right) \operatorname{sgn}\left(\alpha_{i+2^{S-1}}\right) \min \left(\alpha_{i}, \alpha_{i+2^{S-1}}\right), \\
& \alpha_{i}^{r}=\alpha_{i+2^{S-1}}+\left(1-2 \beta_{i}^{l}\right) \alpha_{i} .
\end{aligned}
$$

The LLRs at the root node are initialized as the channel LLR $\boldsymbol{y}_{0}^{N-1}=\left\{y_{0}, y_{1}, \ldots, y_{N-1}\right\}$. Nodes receive the partial sums $\boldsymbol{\beta}$ from their left $\boldsymbol{\beta}^{\boldsymbol{l}}=\left\{\beta_{0}^{l}, \beta_{1}^{l}, \ldots, \beta_{2^{S-1}-1}^{l}\right\}$ and right $\boldsymbol{\beta}^{\boldsymbol{r}}=$ $\left\{\beta_{0}^{r}, \beta_{1}^{r}, \ldots, \beta_{2^{S-1}-1}^{r}\right\}$ child node:

$$
\beta_{i}= \begin{cases}\beta_{i}^{l} \oplus \beta_{i}^{r}, & \text { if } i \leq 2^{S-1} \\ \beta_{i-2^{S-1}}^{r}, & \text { otherwise. }\end{cases}
$$

where $\oplus$ is the bitwise $\mathrm{XOR}$ operation, and $0 \leq i<2^{S}$. At leaf nodes, the $\beta$ value and the estimated bit vector $\hat{\boldsymbol{u}}_{\mathbf{0}}^{N-1}$ are computed as

$$
\beta_{i}= \begin{cases}0, & \text { when } \alpha_{i} \geq 0 \text { or } i \in \Phi \\ 1, & \text { otherwise. }\end{cases}
$$

where $\Phi$ denotes the set of frozen indices.

\section{B. Successive-Cancellation Flip Decoding}

The SC-Flip algorithm was first introduced in [9] to improve finite-length error-correction performance of polar codes. It was observed that when SC decoding fails, it is due to one or more incorrect bit estimations at one of the leaf nodes, that are in fact propagated through the tree due to sequential nature of SC, resulting in more incorrect estimations. As a result, incorrect bit decisions are classified into two categories: errors due to channel noise, and errors caused by a previous incorrect estimation. Consequently, the first observed error is always due to channel noise. Fig. 3 depicts the frequency of occurrence for channel-induced errors, for $P C(512,256)$ with different $E_{b} / N_{0}$ points. It can be observed that most of the decoding failures are due to a single channel-induced error, whose frequency increases with $E_{b} / N_{0}$. On the other hand, Fig. 4 highlights that failures due to a single error are more prominent at target FER $=10^{-4}$, and is observed to be around $90 \%$ for code rates $R \in\left\{\frac{1}{4}, \frac{1}{3}, \frac{1}{2}, \frac{2}{3}, \frac{3}{4}\right\}$. Throughout the rest of this work, the occurrence of a single channel-induced error is denoted as $E_{1}$.

In order to observe the impact of $E_{1}$ on the error-correction performance, a genie-like decoder called SC-Oracle was introduced in [9]: it has foreknowledge of the transmitted 


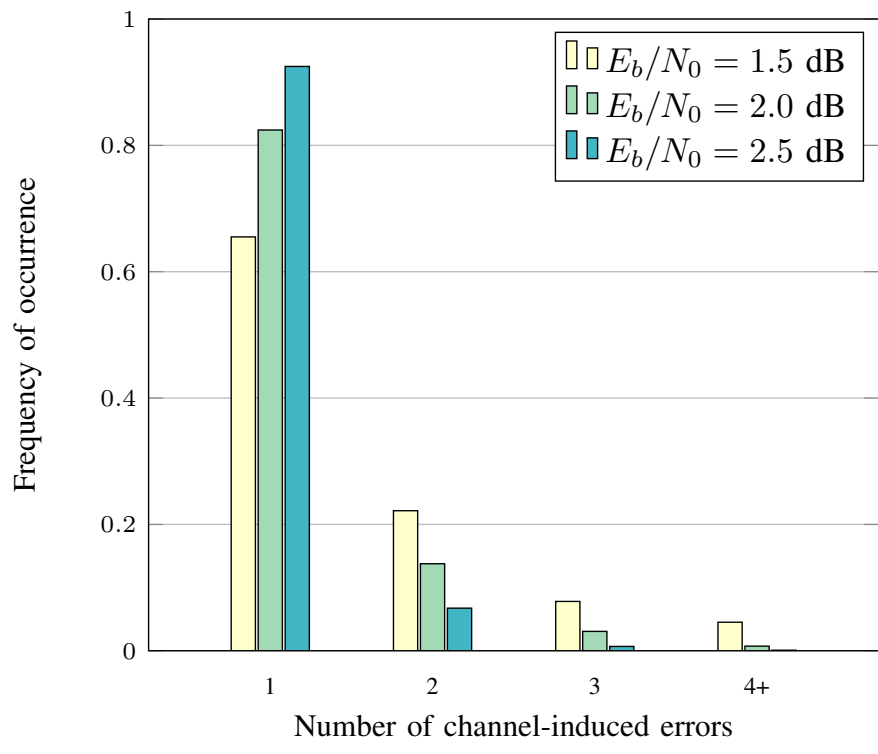

Fig. 3: Frequency of occurrence of channel-induced errors at various $E_{b} / N_{0}$ points for $P C(512,256)$.

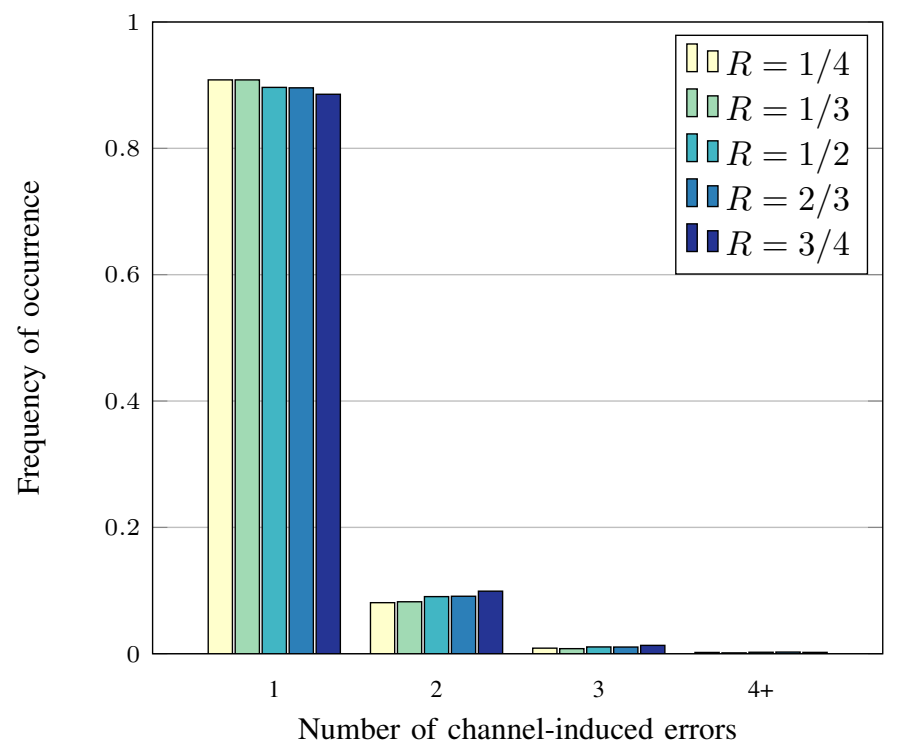

Fig. 4: Frequency of occurrence of channel-incurred errors for polar code with $N=512, R \in\left\{\frac{1}{4}, \frac{1}{3}, \frac{1}{2}, \frac{2}{3}, \frac{3}{4}\right\}$ at target FER $=10^{-4}$.

codeword, which it uses to identify the first channel-induced error and ensure that $\mathrm{SC}$ estimates the bit correctly. In this work, we target the correction of $E_{1}$, and thus consider SCOracle correcting only $E_{1}$ as a baseline. We refer to the corresponding frame error rate (FER) performance as SCO-1 performance. Original SC-Flip algorithm [9] uses a cyclicredundancy check $(\mathrm{CRC})$ code with a $C$-bit remainder to encode the information bits. If the CRC is successful at the end of SC decoding, the estimated codeword is assumed to be correct. In case the CRC fails, the $T_{\max }-1$ LLRs with the smallest magnitude, representing the bit estimations with lowest reliability, are stored and sorted, after which a set of SC decoding attempts are initiated. At each decoding attempt, one of the selected bits is flipped, in ascending reliability order, and the $\mathrm{CRC}$ is checked at the end of each iteration. This process continues for $T_{\max }$ attempts, or until the CRC passes. It should be noted that the initial SC decoding is counted as the first iteration towards $T_{\max }$ maximum iterations.

Since decoding of a single codeword can take up to $T_{\max }$ iterations, the decoding latency of SC-Flip is not fixed. As $T_{\max }$ increases, the error-correction performance of SC-Flip decoding improves towards its lower bound, which is the SCO1 performance with $K+C$ non-frozen bits. The performance gap between SC-Flip and SCO-1 is due to two possible cases: either the estimated codeword with a successful CRC check results in undetected errors, or the decoding stopped after reaching $T_{\max }$ iterations without correcting the error.

Improvements for SC-Flip have been proposed recently in [10] and [11], where a generalized SC-Flip decoding algorithm is used to correct more than one erroneous hard decision through nested flips. A simulation-based scaling metric is also introduced in order to help the SC-Flip decoder detect the erroneous bit indices more accurately. Simulation results show an improvement of $0.4 \mathrm{~dB}$ in error-correction performance when $T_{\max }=10$ with respect to SC-Flip. Practical implementation of this approach requires either a $T_{\max }$ value that is larger by an order of magnitude, or parallel SC-Flip decoders similar to SC-List decoding. Partitioned SC-Flip (PSCF) decoding was introduced in [14], in which the CRC is distributed into multiple partitions in the codeword, in order to improve the error-correction performance and reduce average number of iterations significantly. The first hardware implementation of baseline SC-Flip decoder was recently implemented in a multidecoder chip with $28 \mathrm{~nm}$ CMOS technology which supports configurable CRC length and $T_{\max }$ [16]. Results show that SC-Flip decoding with $C=8$ and $T_{\max }=8$ is up to $32.4 \%$ faster than SC-List at target FER of $10^{-2}$, while its energy consumption is similar to that of an SC decoder.

\section{Improving SC-Flip Based on Error Distribution}

In [12] we performed a simulation campaign with SCOracle, to identify the bit indices more likely to incur in $E_{1}$. We observed that, although all non-frozen indices are theoretically susceptible to channel-induced errors, a fraction of them is more likely to incur one than the others. Thus, only those that bring a substantial contribution to the FER are considered as possibly erroneous. These observations allow to reduce the set of bits to be flipped, and two bit-flipping index selection criteria for SC-Flip have been proposed. The first one, called fixed-index selection (FIS), replaces the selection and sorting process of SC-Flip with a list of critical indices that is pre-sorted based on their $E_{1}$ occurrence frequency. The second method, called enhanced index selection (EIS), applies the LLR-based index selection of SC-Flip to the critical set only, thus excluding indices with a low probability of $E_{1}$. Simulations have shown that there is no error-correction performance loss when FIS is applied to SC-Flip for low-rate 


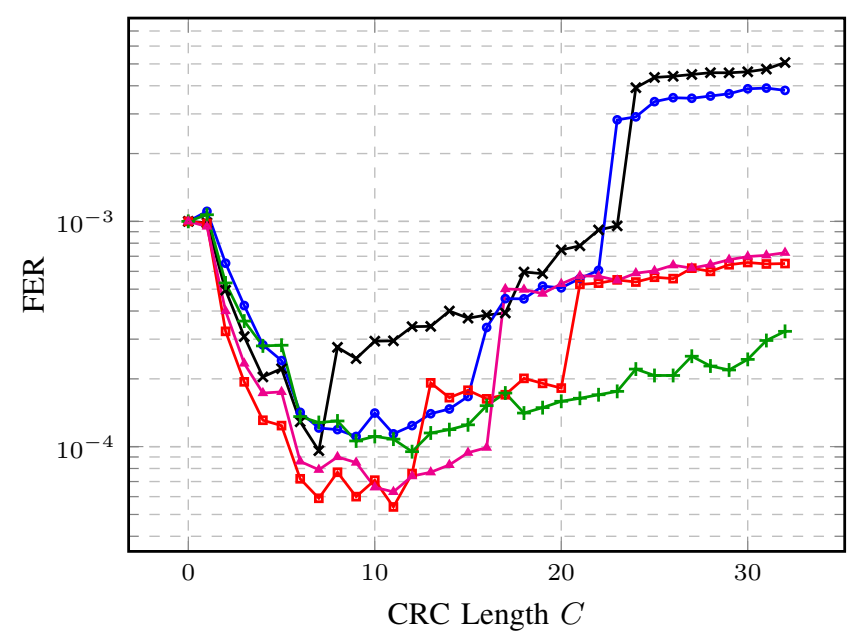

* $P C(1024,170) \rightarrow P C(1024,256) \rightarrow P C(1024,340)$ $\longrightarrow P C(1024,512) \rightarrow P C(1024,680)$

Fig. 5: Error correction performance of SC-Flip decoding with respect to CRC length $C$, for polar codes of various rates.

polar codes, while the LLR sorting needed by the original index selection is avoided, which represents an estimated $24.6 \%$ of the total logic complexity in ASIC implementations. Simulation results for the EIS criterion show gains with respect to SC-Flip of up to $0.4 \mathrm{~dB}$ and $0.42 \mathrm{~dB}$ for $P C(1024,170)$ and $P C(1024,256)$ respectively, that decrease at higher code rates.

A similar approach has been recently taken in [13] to improve the error-correction performance of SC-Flip. A set of indices is created from each sub-tree of the polar code that is a Rate-1 node (i.e. nodes without any frozen bits), including almost all channel-induced errors. The size of the index set increases with $E_{b} / N_{0}$, and can be used progressively to correct multiple errors, achieving an error-correction performance comparable to that of SC-List with $L=32$. However, a very high $T_{\max }$ is needed by this technique, since the bit flipping search space must include the entire set; which also leads to very long worst-case latency.

\section{Thresholded IndeX SELECTION FOR SC-FliP DECODING}

The FIS and EIS criteria [12] observe the distribution of $E_{1}$ occurrences to limit the set of indices for bit flipping to reduce the implementation complexity and improve the errorcorrection performance of SC-Flip. However, their benefits diminish at higher code rates. In this Section, we use the SCOracle decoder to provide further insight about failed decoding attempts, and propose a new index selection scheme for SCFlip. We detail the proposed method focusing on low-rate codes, and extend it to higher-rate polar codes in Section III-C. The CRC remainder length for each rate is selected with respect to the FER observation in Fig. 5; it can be seen that the best $C$ value is different for each rate. The polar code used in this work is constructed targeting an $E_{b} / N_{0}$ value of $2.5 \mathrm{~dB}$, using [15]. The distribution of $E_{1}$, the critical set, and the optimal threshold values may change with a different polar code construction, but the proposed approach is independent of it.

As mentioned in Section II-C, all non-frozen indices are theoretically susceptible to channel-induced errors. If the maximum number of iterations is large enough to flip all possibly erroneous bits, i.e. $T_{\max }=K+C$, and assuming a perfect CRC, SC-Flip can identify and correct all $E_{1}$ occurrences and achieve the SCO-1 performance. Let us define the set of non-frozen indices as $\mathcal{I}^{N F}$, and their associated frequency of $E_{1}$ occurrence as $f^{E_{1}}$. For example, consider the polar code $P C(8,5)$ in Fig. 2. The non-frozen indices are $\mathcal{I}^{\boldsymbol{N} \boldsymbol{F}}=\left\{u_{3}, u_{4}, u_{5}, u_{6}, u_{7}\right\}$, and assume $\boldsymbol{f}^{\boldsymbol{E}_{1}}=$ $\{0.25,0.40,0.20,0.13,0.02\}$, respectively. In this situation, the most critical index is $u_{4}$, followed by $u_{3}$, and so on. Note that the summation of all the elements in $f^{E_{1}}$ is equal to 1 . Considering the whole $\mathcal{I}^{N F}$ for bit-flipping is not feasible for practical applications since the maximum and average number of iterations would be extremely large. Fig. 6 depicts $f^{E_{1}}$ for all non-frozen bits in $P C(1024,170)$ with $C=32$ at two different $E_{b} / N_{0}$ values. As the simulations to identify the frequency of $E_{1}$ occurrence have to run for a finite time, a target FER $\left(\mathrm{FER}_{\mathrm{t}}\right)$ is required to determine the minimum amount of simulated frames. In this work, we set $\mathrm{FER}_{\mathrm{t}}$ as $10^{-4}$. It can be seen that with the target $\mathrm{FER}_{\mathrm{t}}$, only a limited set of non-frozen indices has non-negligible $f^{E_{1}}$. Let us define the FER obtained with SCO- 1 at a certain $E_{b} / N_{0}$ as FER $_{\mathrm{SCO}-1}$ and the FER obtained with SC at the same $E_{b} / N_{0}$ point $\left(\mathrm{FER}_{\mathrm{SC}}\right)$. Then:

$\sum f_{i}^{E_{1}} \times\left(\mathrm{FER}_{\mathrm{SC}}-\mathrm{FER}_{\mathrm{SCO}-1}\right)=\mathrm{FER}_{\mathrm{SC}}-\mathrm{FER}_{\mathrm{SCO}-1}$,

where $f_{i}^{E_{1}}$ is the $i^{\text {th }}$ element of $\boldsymbol{f}^{E_{1}}$. This shows that considering separately all the contributions to FER ${ }_{S C}$ of $E_{1}$ and summing them together, we get as a result the difference between FER ${ }_{\text {SC }}$ and FER ${ }_{\text {SCO-1 }}$. The critical set $\mathcal{I}^{C}$ is defined as the smallest subset of $\mathcal{I}^{N F}$ that satisfies the following:

$\sum f_{i}^{E_{1}} \times\left(\mathrm{FER}_{\mathrm{SC}}-\mathrm{FER}_{\mathrm{SCO}-1}\right) \geq \gamma \times\left(\mathrm{FER}_{\mathrm{SC}}-\mathrm{FER}_{\mathrm{SCO}-1}\right)$,

where $\gamma \approx 1$. This means that if we exclude some of the non-frozen bit indices from the previous calculation, we can identify a factor $\gamma \approx 1$ so that the summation of the considered $f_{i}^{E_{1}}$ is equal to $\gamma$, and the consequent difference in $\mathrm{FER}_{\mathrm{SC}}-\mathrm{FER}_{\mathrm{SCO}-1}$, and thus FER degradation, is negligible. In the remainder of this work, $\gamma$ is set to 0.9999 . The nonfrozen indices belonging to $\mathcal{I}^{C}$ are called critical indices.

Additional insight on the behavior of erroneous indices can be gained by observing the magnitude of LLRs. Fig. 7 plots the average LLR magnitude for $\mathcal{I}^{\boldsymbol{N} \boldsymbol{F}}$ of $P C(1024,170)$, normalized with the maximum LLR value: it can be seen that all of the error-prone indices identified in Fig. 6, highlighted in red in here, are associated to small average LLR magnitudes. Fig. 8 depicts an example of average LLR magnitudes for the indices in the critical set $\mathcal{I}^{C}$, for the case in which an $E_{1}$ occurs at the corresponding index (in red) and for when it does not (in blue). The average LLR magnitudes are substantially larger in case of successful decoding, compared to when it fails.

Once the critical set $\mathcal{I}^{C}$ has been identified, the trend observed in Fig. 8 can be used to apply further refinement 


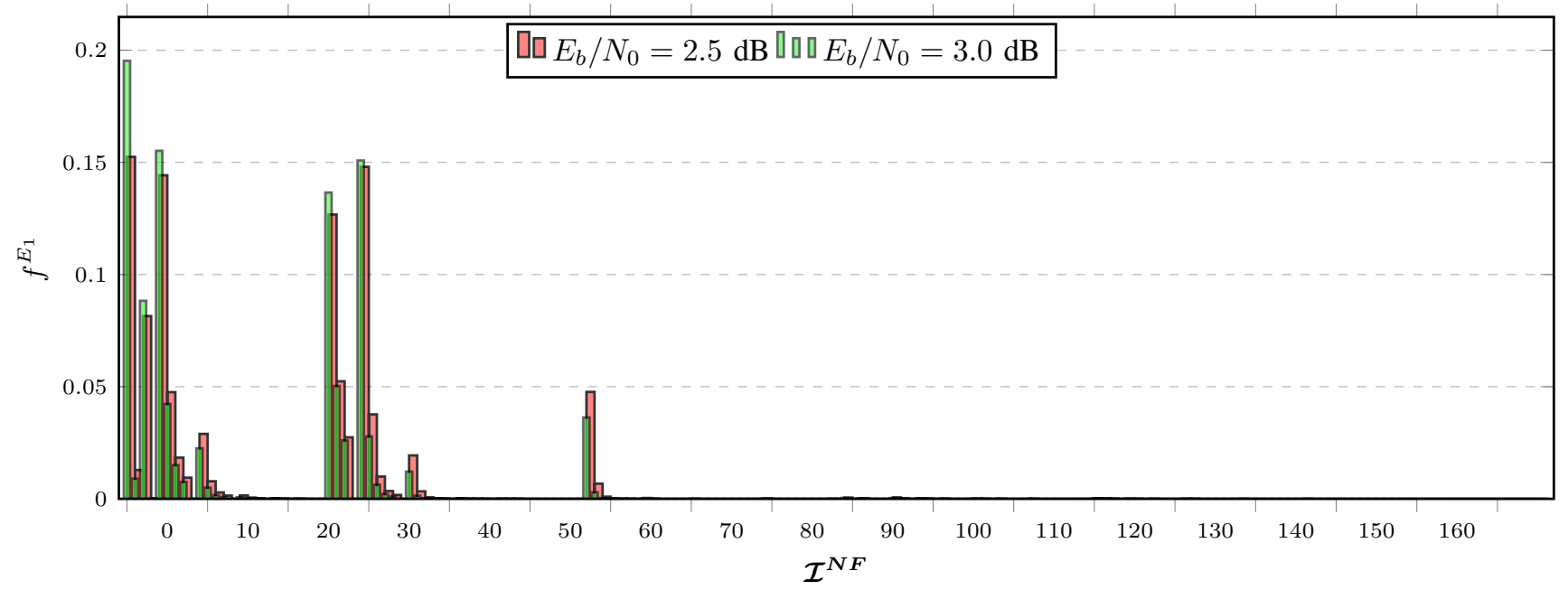

Fig. 6: $f^{E_{1}}$ values corresponding to each non-frozen index of $P C(1024,170), C=7, \mathrm{FER}_{\mathrm{t}}=10^{-4}$.

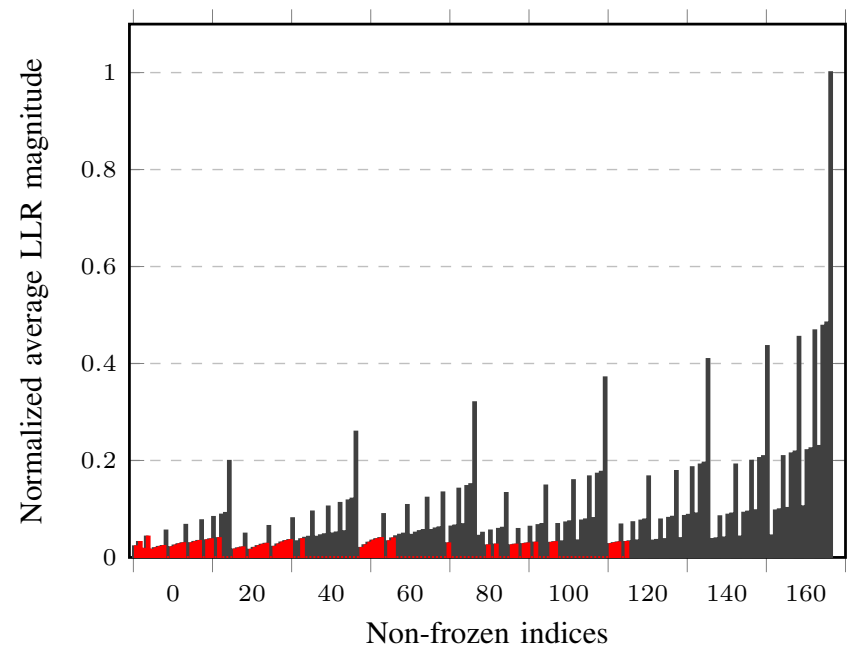

Fig. 7: Normalized average LLR magnitude at each non-frozen bit index for $P C(1024,170)$ at $E_{b} / N_{0}=2.5 \mathrm{~dB}$ and $C=7$, $\mathrm{FER}_{\mathrm{t}}=10^{-4}$. Indices highlighted in red correspond to the non-zero $f^{E_{1}}$ shown in Fig. 6 .

to the bit-flipping search space through an LLR threshold. Critical indices are considered to be possibly in error only if their LLR is lower than the threshold. We call this technique thresholded index selection (TIS), while SC-Flip incorporating TIS is identified as thresholded SC-Flip (TSCF). After TIS, the identified indices are flipped starting from the leftmost one. This is due to the fact that LLR magnitudes are affected by the propagation of errors, and a small LLR can be caused by a previous channel-induced wrong decision. Thus, selecting the $T_{\max }$ indices with the lowest LLR magnitude, like in the original SC-Flip [9], would include critical indices that have a lower $f^{E_{1}}$, but have been adversely affected by the earlier wrong decision. This approach gives superior errorcorrection performance with respect to the LLR-based sorting, and substitutes the sorter with a low-complexity threshold comparator.

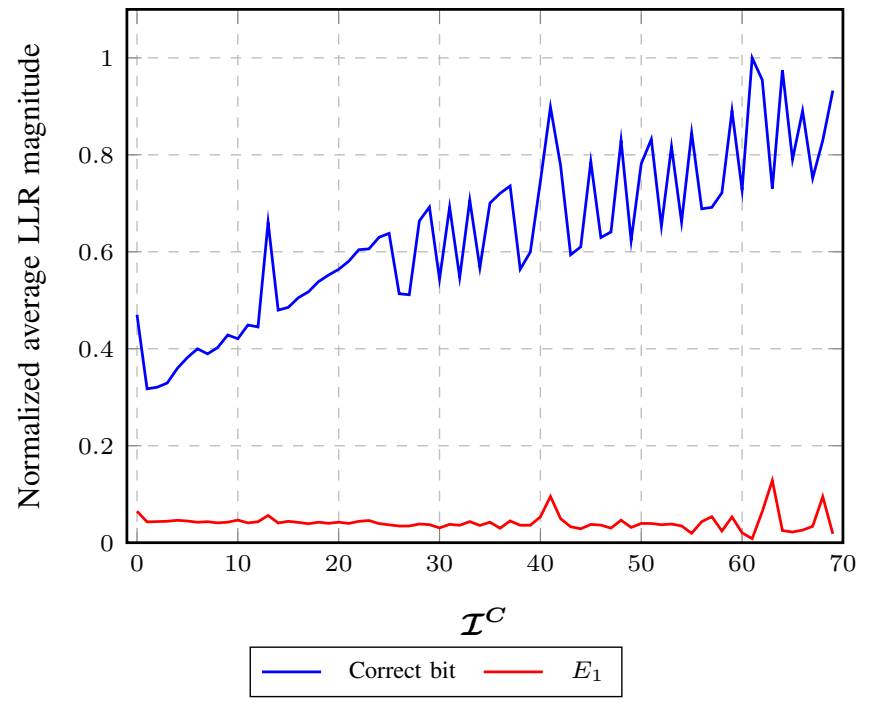

Fig. 8: Normalized average LLR magnitudes for $\mathcal{I}^{C}$, $P C(1024,170), C=7$ at $E_{b} / N_{0}=2.5 \mathrm{~dB}$. Correct bit estimation is in blue, $E_{1}$ occurrence is in red.

\section{A. LLR Threshold Selection}

The effectiveness of TIS and TSCF is based on the significant difference in magnitude between the average LLR values of the critical indices in case of successful and failed decoding, that allows the identification of an LLR threshold. Let us identify this threshold as $\Omega$. In order to maximize the accuracy of the thresholding process, the distribution of LLR values for critical indices needs to be observed.

While Fig. 8 depicts the LLR magnitude gap between the average LLR magnitudes for $E_{1}$ occurrence and correct bit estimation, Fig. 9 shows the distributions of LLR magnitudes for the first index in the critical set $\mathcal{I}_{0}^{C}$ in case $E_{1}$ occurred there (red line) and when it did not (blue line). The vertical line in Fig. 9 shows where the two distributions overlap, and represents an ideal thresholding point. In fact, it is very likely that LLRs to the left of the line represent erroneous decisions, 


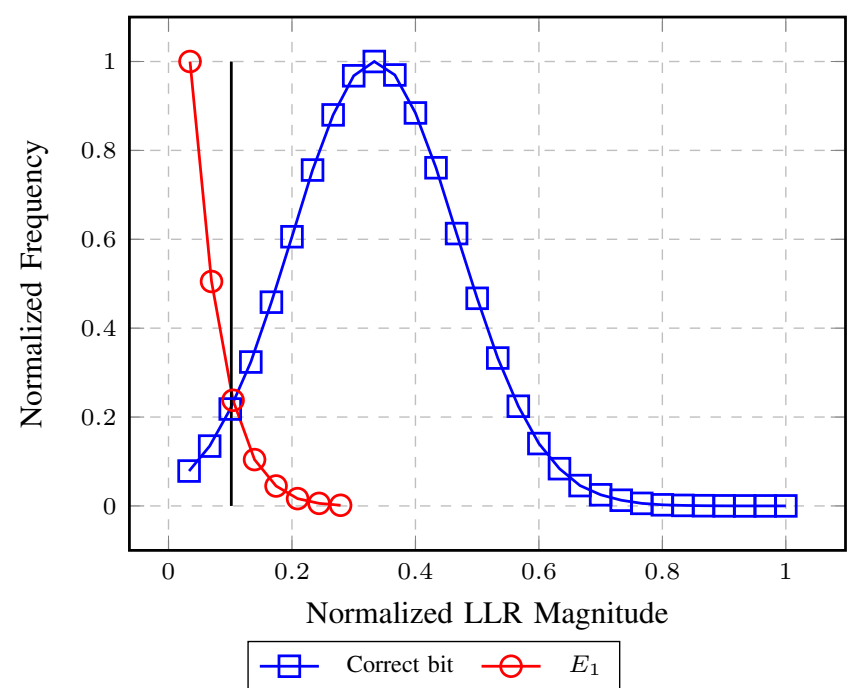

Fig. 9: Distribution of LLR magnitude in case of correct bit estimation (blue) and $E_{1}$ (red) for critical index $\mathcal{I}_{0}^{C}$ for $P C(1024,170)$ and $C=7$ at $E_{b} / N_{0}=3.25 \mathrm{~dB}$.

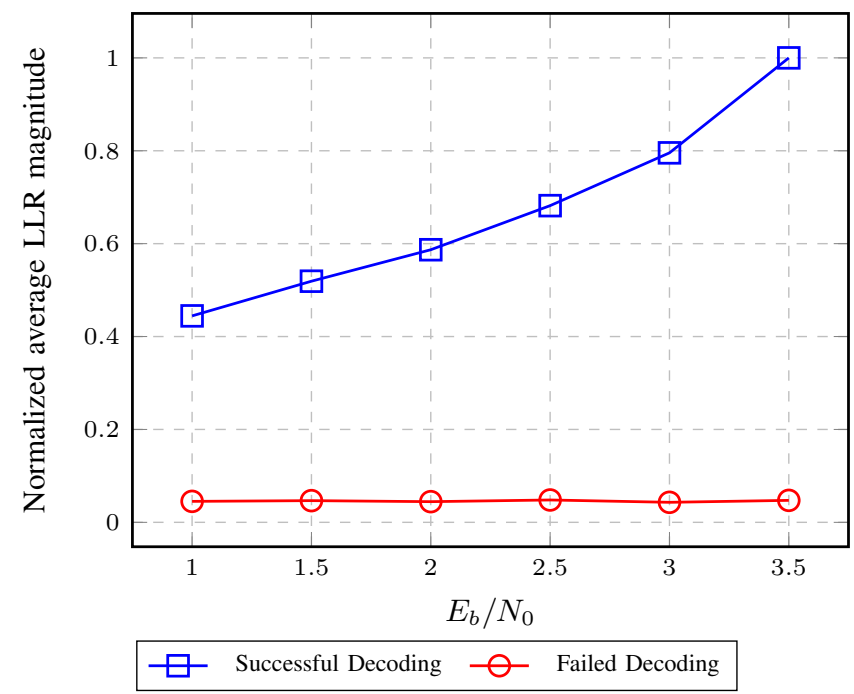

Fig. 10: Normalized LLR magnitude averaged over the whole $\mathcal{I}^{C}$ with respect to $E_{b} / N_{0}$, in case of successful (blue) and failed (red) decoding; $P C(1024,170)$ and $C=7$.

while the LLRs to its right are very likely to represent a correct estimation.

A possible approach to thresholding is to set a different $\Omega$ for each index at the crossover point between the correct and erroneous LLR distributions. However, this approach results in large memory consumption and the threshold list depends on the size of $\mathcal{I}^{C}$. It can however be observed in Fig. 8 that while the mean of correct LLRs varies with each critical index, the mean of erroneous LLRs remains very low. Consequently, for a target $E_{b} / N_{0}$, a single threshold for entire set $\mathcal{I}^{C}$ can be selected. In Section IV, it is shown that TSCF with a single threshold for the whole critical set is sufficient to approach the SCO-1 performance within a small number of iterations.

Average LLR magnitudes do not only depend on the critical index, but also on $E_{b} / N_{0}$. A similar trend is in fact observed

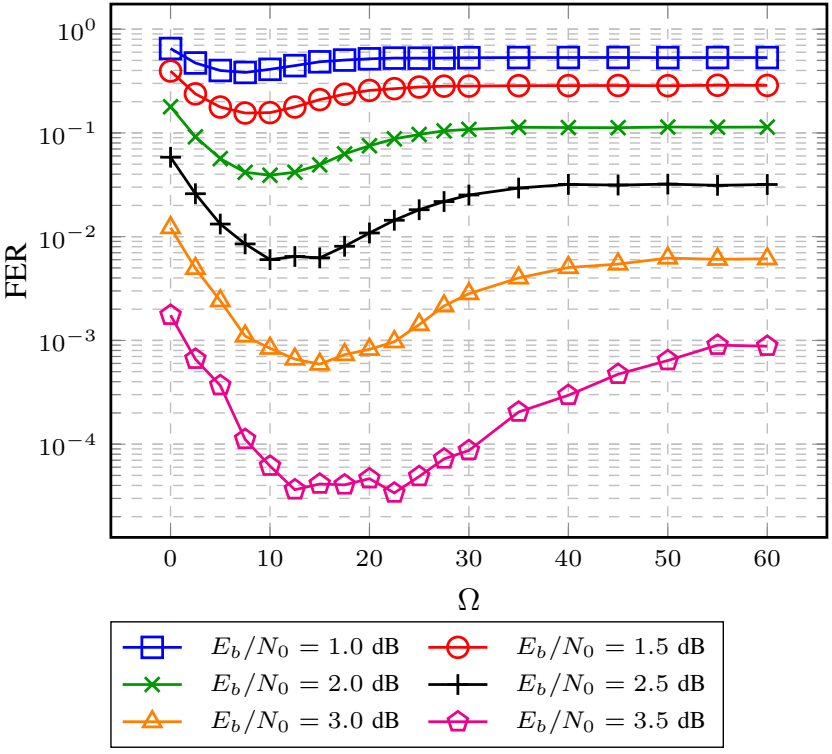

Fig. 11: FER curves for TSCF decoding with various $\Omega$ values for $P C(1024,170), C=7, T_{\max }=10$.

in Fig. 10, where it is shown that while the average LLRs for critical indices tend to increase with $E_{b} / N_{0}$ in case of correct estimations, the erroneous LLR average remains around the same magnitude, resulting in an increasing gap between them. As a result, there is a different threshold value for each $E_{b} / N_{0}$ that maximizes the error-correction performance gain of TSCF. Based on the observations from Fig. 8, Fig. 9 and Fig. 10, an LLR threshold sweep is performed for TSCF decoding. Fig. 11 presents the FER of $P C(1024,170)$ for various $E_{b} / N_{0}$ values for a set of $\Omega$ values, with $C=7$ and $T_{\max }=10$. The critical set $\mathcal{I}^{C}$ is obtained at $E_{b} / N_{0}=3.0 \mathrm{~dB}$ and includes 56 indices. The best FER can be obtained by selecting a different threshold value for each $E_{b} / N_{0}$. However, if a single LLR threshold for all $E_{b} / N_{0}$ points is more suitable for the application, it should be selected in accordance with the target FER. It can be seen that the $\Omega$ value that corresponds to best FER value tends to increase as the channel conditions improve. Also note that, with $\Omega=0$, the LLR values of critical indices will always fall above the threshold, and TSCF reverts to SC decoding with $K+C$ non-frozen bits.

Based on the information obtained on the impact of $\Omega$ on FER, TSCF has been compared to the baseline SC-Flip algorithm. Fig. 12 shows the change in FER with respect to $T_{\max }$ for both algorithms at various $E_{b} / N_{0}$ values. The same critical set used in Fig. 11 is used here. Solid curves represent TSCF with the optimal $\Omega$ value for each $E_{b} / N_{0}$, and dashed lines represent SC-Flip. Simulations are performed for $P C(1024,170)$ for up to maximum $T_{\max }$ of 20 . It can be seen that while the error-correction performance of SC-Flip improves almost linearly with $T_{\max }$, TSCF quickly converges to a lower bound in each case, i.e. the SCO-1 performance. This is due to the fact that TSCF is able to find the correct critical index quicker than SC-Flip. As a result, TSCF can reach a target FER with a smaller $T_{\max }$ value, which results in a shorter worst case decoding latency and more stable average 


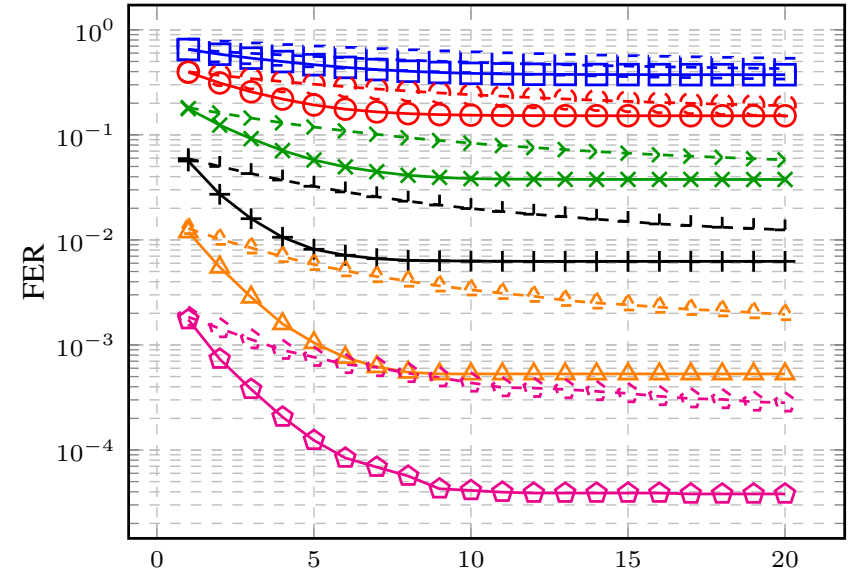

$T_{\max }$

\begin{tabular}{|c|c|c|c|}
\hline$\square$ & $E_{b} / N_{0}=1.0$ & $-\stackrel{E}{-2}$ & $E_{b} / N_{0}=1.0 \mathrm{~dB}$ \\
\hline 0 & $E_{b} / N_{0}=1.5 \mathrm{~dB}-\Omega=7.5$ & $-\leq L$ & $E_{b} / N_{0}=$ \\
\hline$\leftarrow$ & $\mathrm{dB}-\Omega=10$ & $-\rightarrow-$ & $0=2.0 \mathrm{~dB}$ \\
\hline+ & $E_{b} / N_{0}=$ & $-\lrcorner-$ & $T_{0}=2.5 \mathrm{~dB}$ \\
\hline$\triangle$ & $E_{b} / N_{0}=$ & $-\underline{-}-$ & $E_{b} / N_{0}=$ \\
\hline 定 & $E_{b} / N_{0}=3.5 \mathrm{~dB}-\Omega=22.5$ & -ミ- & $E_{b} / N_{0}=3.5 \mathrm{~dB}$ \\
\hline
\end{tabular}

Fig. 12: FER curves for TSCF decoding (solid lines) and SC-Flip (dashed lines) with various $T_{\max }$ values, for $P C(1024,170), C=7$.

latency. For example, it takes only 5 iterations for TSCF to achieve FER $=10^{-3}$ at $E_{b} / N_{0}=3.0 \mathrm{~dB}$, while it takes 20 iterations for SC-Flip. From another point of view, given a fixed maximum number of iterations $T_{\max }>1$, TSCF results in lower FER than SC-Flip in all cases. For example. with $T_{\max }=5$, TSCF has FER $=1.23 \times 10^{-4}$ at $E_{b} / N_{0}=3.5$ $\mathrm{dB}$, where it is $7.65 \times 10^{-4}$ for SC-Flip. Finally, note the crossing of the SC-Flip curve at $E_{b} / N_{0}=3.5 \mathrm{~dB}$ with TSCF at $E_{b} / N_{0}=3.0 \mathrm{~dB}$. This crossover means that, at $T_{\max } \in\{6,7,8\}$, both SC-Flip and TSCF have identical errorcorrection performance; however TSCF achieves this while having an $E_{b} / N_{0}$ value that is $0.5 \mathrm{~dB}$ less than that of SCFlip.

\section{B. Critical Set Selection}

The identification of $\mathcal{I}^{C}$ using SC-Oracle helps improve error-correction performance and reduce $T_{\max }$. However, not every critical index in Fig. 6 has the same $f^{E_{1}}$. In fact given FER $_{\mathrm{t}}$, at each $E_{b} / N_{0}$ SC-Oracle reports $\mathcal{I}^{C}$ of different sizes and composed of different indices. For example, in Fig. 6, although the 6 indices with the largest $f^{E_{1}}$ are the same for considered $E_{b} / N_{0}$ points, the index with $7^{\text {th }}$ largest $f^{E_{1}}$ is different. It should be also noted that SC-Oracle identifies a smaller list of indices as $E_{b} / N_{0}$ grows, which is a subset of that identified for lower $E_{b} / N_{0}$ values.

It can be seen in Fig. 6 that the majority of $f^{E_{1}}$ is concentrated around a small set of non-frozen indices. For example, the first seven indices that are most likely to incur an error account for $75.3 \%$ of $f^{E_{1}}$. As we addressed in Section III, it is thus unnecessary to consider all indices that have nonzero $f^{E_{1}}$ as part of the critical set, especially considering

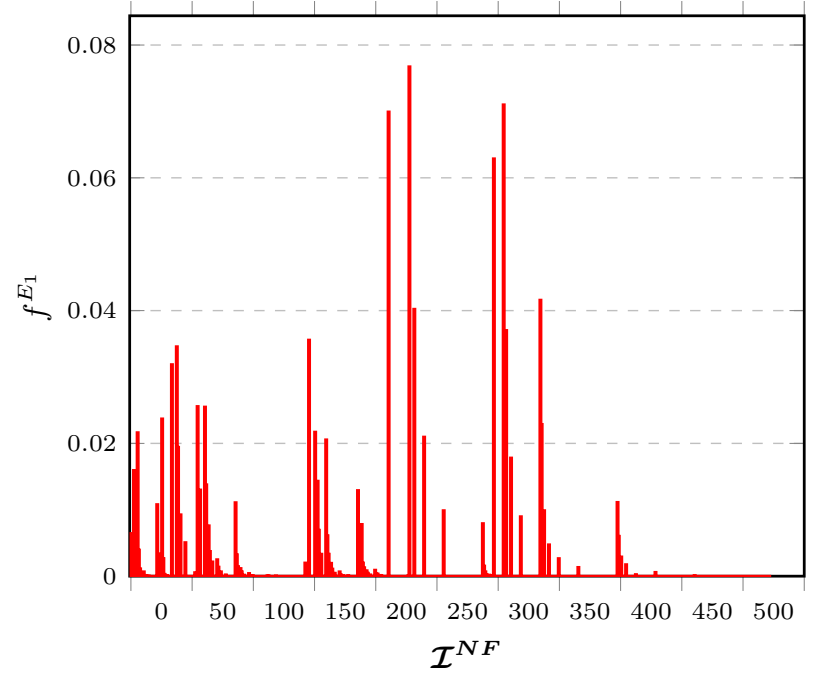

Fig. 13: $f^{E_{1}}$ values for non-frozen indices of $P C(1024,512)$, $C=12, E_{b} / N_{0}=3.0 \mathrm{~dB}$.

the limits imposed by $T_{\max }$. A large critical set, together with a small $T_{\max }$, can lead to substantial error-correction performance degradation. Moreover, in order to minimize the TSCF decoder implementation cost, a single critical set is desirable. A first critical set selection approach is to use the $\mathcal{I}^{C}$ derived from the lowest considered $E_{b} / N_{0}$, given the fact that at higher $E_{b} / N_{0}$ the critical set is a subset of this one. With a high enough $T_{\max }$, such a set can correct all $E_{1}$ at higher $E_{b} / N_{0}$ values, achieving the SCO-1 performance. On the other hand, some of the critical indices only occur at low $E_{b} / N_{0}$ values, introducing unnecessary latency and memory usage for iterations at high $E_{b} / N_{0}$ points. Thus, a more efficient approach selects the set of indices starting from the full set identified for a high $E_{b} / N_{0}$ value. For example, out of the 56 critical indices identified after omitting the ones with low $f^{E_{1}}$ for $P C(1024,170)$ with $C=7$ at $E_{b} / N_{0}=3.0 \mathrm{~dB}$, all of them exist within the 70 indices found at $E_{b} / N_{0}=2.5 \mathrm{~dB}$, and they are within the most critical ones. Similarly, they in turn constitute a subset of the 81 indices found at $E_{b} / N_{0}=2.0 \mathrm{~dB}$. Finally, where all the indices of $E_{b} / N_{0}=3.0 \mathrm{~dB}$ represent $100 \%$ of all $E_{1}$ occurrences, the same set of indices covers $99.99 \%$ and $99.96 \%$ of all $E_{1}$ errors for when $E_{b} / N_{0}=2.5$ and $2.0 \mathrm{~dB}$, respectively. As a result, a single $\mathcal{I}^{C}$ identified at a high $E_{b} / N_{0}$ value can be selected with negligible degradation in error-correction performance at lower $E_{b} / N_{0}$ values.

It should be noted that the discussed critical index selection scenarios can also be applied to the FIS and EIS criteria described in [12]. Finally, the indices and size of the critical set may change with respect to polar code construction.

\section{TSCF Decoding for Higher Rate Polar Codes}

The advantages of TSCF decoding can also be observed in high and medium code rates. With increased rate, the size of $\mathcal{I}^{C}$ increases, making it more difficult to identify the correct flipping index for both TSCF and SC-Flip decoding. Finally, $f^{E_{1}}$ becomes more distributed over the whole codeword, as 


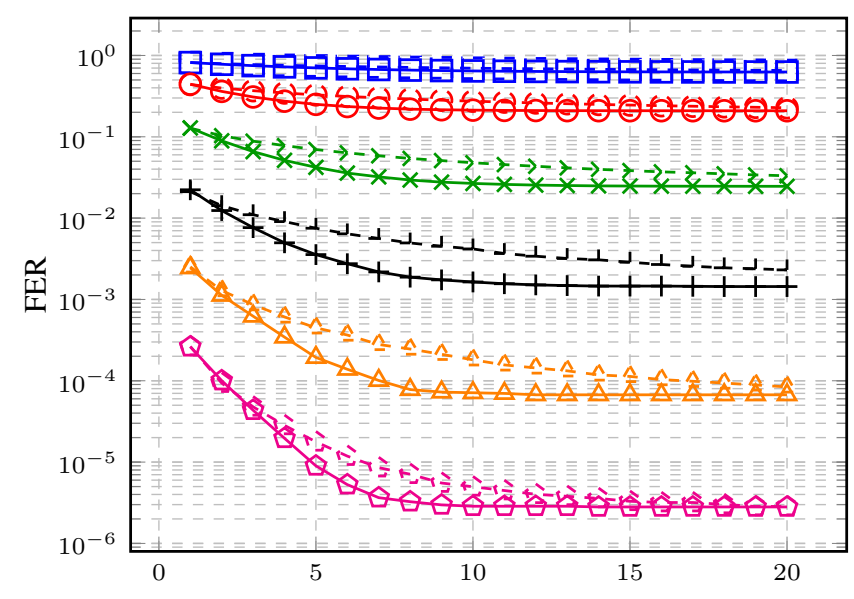

$T_{\max }$

\begin{tabular}{|c|c|c|c|}
\hline$\square$ & $E_{b} / N_{0}=1.0 \mathrm{~dB}-\Omega=5$ & - İ- & $E_{b} / N_{0}=1.0 \mathrm{~dB}$ \\
\hline$\vartheta$ & $E_{b} / N_{0}=1.5 \mathrm{~dB}-\Omega=5$ & $-\leqslant 2$ & $E_{b} /$ \\
\hline$*$ & $E_{b} / N_{0}=2.0 \mathrm{~dB}-\Omega=7.5$ & $-\rightarrow-$ & $E_{b} /$ \\
\hline+ & $E_{b} / N_{0}=2$ & $-\lrcorner$ & $E_{b} / N_{0}=2.5 \mathrm{~dB}$ \\
\hline$\Delta$ & $E_{b} / N_{0}=3.0 \mathrm{~dB}-\Omega=12.5$ & $-\leq-$ & $E_{b} / N_{0}=3.0 \mathrm{~dB}$ \\
\hline 合 & $E_{b} / N_{0}=3.5 \mathrm{~dB}-\Omega=15$ & $-\leftrightarrows-$ & $E_{b} / N_{0}=3.5 \mathrm{~dB}$ \\
\hline
\end{tabular}

Fig. 14: FER curves for TSCF decoding (solid lines) and SC-Flip (dashed lines) with various $T_{\max }$ values, for $P C(1024,512), C=12$.

depicted in Fig. 13 for $P C(1024,512)$. Due to these reasons, the average number of iterations $T_{a v g}$ increases with the rate, where a full iteration is considered as $N$ bits being decoded. Consequently, each decoding attempt by SC-Flip or TSCF accounts for a fraction of a full iteration.

Table I details the size of $\mathcal{I}^{C}$ observed for different rates, for various $E_{b} / N_{0}$ points. As mentioned in Section III-B, the total number of critical indices for a fixed rate decreases with increasing $E_{b} / N_{0}$. On the other hand, as the rate increases, a higher number of non-frozen bits are present, and are assigned to less and less reliable channels. Consequently, more indices become susceptible to channel errors. In Table II, cardinality of critical sets with respect to a set of target FER $\left(\mathrm{FER}_{t} \in\right.$ $\left\{10^{-2}, 10^{-3}, 10^{-4}\right\}$ ) for various rates are presented. Similar to Table I, the size of the critical set increases with the code rate in general. On the other hand, as the target FER is improved, the cardinality of critical set decreases for any rate, since the occurrence of channel-induced errors decrease with increased $E_{b} / N_{0}$.

As the impact of $E_{1}$ decreases, the error-correction performance gap between baseline SC-Flip algorithm and SCO1 narrows. As a result, the required $T_{\max }$ value for SC-Flip algorithm to reach to the performance of SCO-1 decreases with increasing rate. In Fig. 14 the FER performance of SC-Flip and TSCF are compared against each other for $P C(1024,512)$ and the optimal $\Omega$ value for each $E_{b} / N_{0}$. As $T_{\max }$ increases, compared to $R=\frac{1}{6}$ (Fig. 12), the FER of both SC-Flip and TSCF converges faster to the lower limit imposed by the SCO1 performance. Nonetheless, TSCF is shown to converge faster than SC-Flip at high rates, yielding comparatively improved error-correction performance and $T_{\max }$.

Table III presents the optimized $\Omega$ values for $N=1024$
TABLE I: Number of critical indices with respect to $E_{b} / N_{0}$ and $R$ for $N=1024$. The optimal CRC length for each rate is chosen from Fig. 5.

\begin{tabular}{|c|c|c|c|c|c|}
\hline \multirow{2}{*}{$\begin{array}{c}E_{b} / N_{0} \\
{[\mathrm{~dB}]}\end{array}$} & \multicolumn{5}{|c|}{ Rate $(R)$} \\
\hline & $1 / 6$ & $1 / 4$ & $1 / 3$ & $1 / 2$ & $2 / 3$ \\
\hline 1.0 & 91 & 118 & 138 & 163 & 154 \\
\hline 1.5 & 89 & 110 & 131 & 160 & 169 \\
\hline 2.0 & 83 & 101 & 125 & 147 & 169 \\
\hline 2.5 & 72 & 85 & 106 & 130 & 155 \\
\hline 3.0 & 56 & 69 & 78 & 113 & 133 \\
\hline 3.5 & 39 & - & - & 74 & 109 \\
\hline 4.0 & - & - & - & - & 71 \\
\hline
\end{tabular}

TABLE II: Number of critical indices with respect to target FER $\left(\mathrm{FER}_{t}\right)$ and $R$ for $N=1024, C=32$.

\begin{tabular}{|c|c|c|c|c|c|}
\hline \multirow[t]{2}{*}{$\mathrm{FER}_{t}$} & \multicolumn{5}{|c|}{ Rate $(R)$} \\
\hline & $1 / 6$ & $1 / 4$ & $1 / 3$ & $1 / 2$ & $2 / 3$ \\
\hline $10^{-2}$ & 64 & 86 & 114 & 131 & 126 \\
\hline $10^{-3}$ & 47 & 71 & 98 & 117 & 107 \\
\hline $10^{-4}$ & 32 & 58 & 76 & 93 & 80 \\
\hline
\end{tabular}

TABLE III: Optimal $\Omega$ values for $N=1024$ and various rates at different $E_{b} / N_{0}$ points.

\begin{tabular}{|c|c|c|c|c|c|}
\hline \multirow{2}{*}{$\begin{array}{c}E_{b} / N_{0} \\
{[\mathrm{~dB}]}\end{array}$} & \multicolumn{5}{|c|}{ Rate $(R)$} \\
\hline & $1 / 6$ & $1 / 4$ & $1 / 3$ & $1 / 2$ & $2 / 3$ \\
\hline 1.0 & 7.5 & 5 & 5 & 5 & 5 \\
\hline 1.5 & 7.5 & 7.5 & 7.5 & 5 & 5 \\
\hline 2.0 & 10 & 10 & 10 & 7.5 & 5 \\
\hline 2.5 & 10 & 10 & 10 & 10 & 5 \\
\hline 3.0 & 15 & 12.5 & 15 & 12.5 & 7.5 \\
\hline 3.5 & 22.5 & - & - & 15 & 10 \\
\hline 4.0 & - & - & - & - & 12.5 \\
\hline
\end{tabular}

and the rates considered in this work. $\Omega$ values are swept with a step size of 2.5. It is observed that $\Omega$ values that provide the best error-correction performance increase with increasing $E_{b} / N_{0}$, since the LLR magnitude gap increases (Fig. 10). On the other hand, it can be observed that the optimal $\Omega$ decreases as the code rate rises. This is due to the fact that the mean LLR magnitude in case of successful decoding decreases with the code rate.

\section{Simulation Results}

In this Section, we evaluate the error-correction performance, the maximum number of iterations $T_{\max }$, and average number of iterations $T_{\text {avg }}$ of TSCF decoding, with respect to SC-Flip, SC-Oracle and SC-List decoding. For all SC-Flip based implementations including TSCF, $C \in\{7,9,9,12,12\}$ for $R \in\left\{\frac{1}{6}, \frac{1}{4}, \frac{1}{3}, \frac{1}{2}, \frac{2}{3}\right\}$ are selected as CRC remainder length, using Fig. 5. While an outer CRC code improves the errorcorrection performance of SC-List decoding significantly at medium to high code rates, it was shown in [17] that at low code rates the CRC is detrimental. Thus, for a fair comparison, we did not consider any CRC in the SC-List curves for low- 


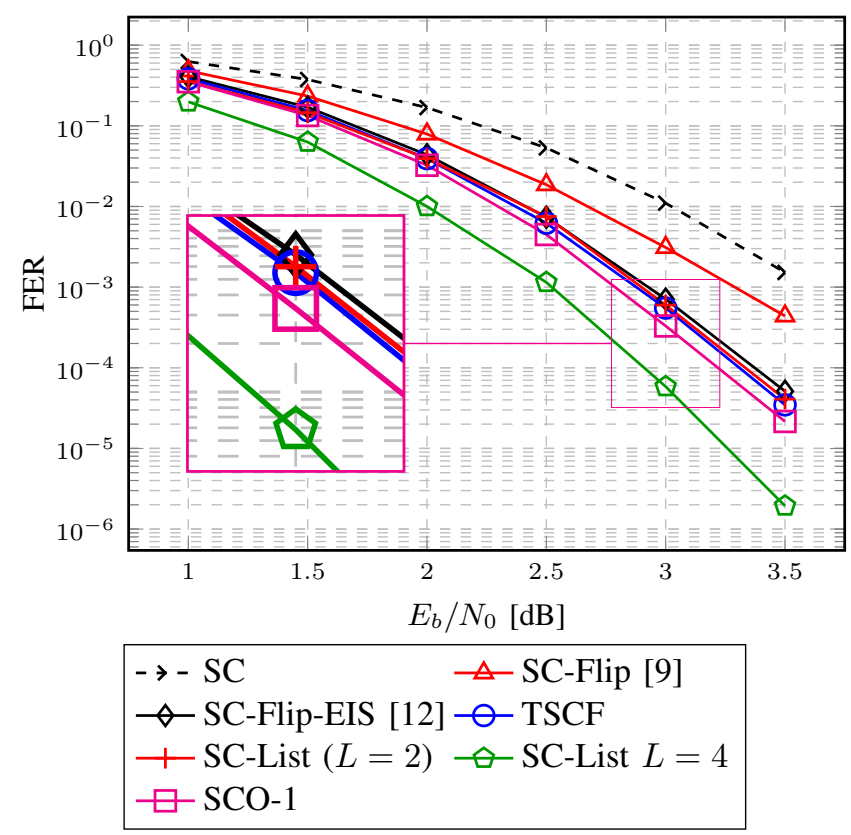

Fig. 15: FER curves with various decoding algorithms for $P C(1024,170) . C=7$ and $T_{\max }=10$ for all SC-Flip based implementations.

rate codes. For the performance comparison of high-rate codes, CRC-aided SC-List is used with $C=8$ [17].

\section{A. Error-Correction Performance}

The error-correction performance of TSCF is compared to SC, SC-Flip, SC-Flip-EIS from [12], SC-List and SCO1. Simulations are performed with binary phase-shift keying (BPSK) modulation and additive white Gaussian noise (AWGN) channel. The same $T_{\max }$ is selected for all SC-Flip based algorithms, the lowest value for which TSCF decoding meets the error-correction performance of SCO-1, as a $T_{\max }$ higher than that will not improve the FER any further. Fig. 15 presents the error-correction performance for $P C(1024,170)$.

Multiple $\Omega$ values are used from Table III in order to optimize the error-correction performance for each $E_{b} / N_{0}$ point. Set $\mathcal{I}^{C}$ for $E_{b} / N_{0}=3.0 \mathrm{~dB}$ from Table I is used. It can be seen that TSCF decoding achieves the SCO-1 performance with $T_{\max }=10$, outperforming SC-Flip decoding with the same $T_{\max }$ value by $0.43 \mathrm{~dB}$ at FER of $10^{-4}$. SC-Flip-EIS decoding from [12] has an error-correction performance that also approaches to SCO-1 performance, but it can be seen that it is slightly worse than that of TSCF. It can also be seen that TSCF decoding approaches SC-List decoding performance with list size $L=2$.

If a single $\Omega$ is allowed regardless of the $E_{b} / N_{0}$ value, it should be selected from Table III to optimize the errorcorrection performance at $\mathrm{FER}_{t}$. Fig. 16 presents the FER of TSCF decoding with single $\Omega$ values. It can be seen that while some $\Omega$ values help TSCF match with SCO- 1 performance at low $E_{b} / N_{0}$, the FER diverges towards SC-Flip performance as $E_{b} / N_{0}$ increases. On the other hand, if an $\Omega$ value for a higher $E_{b} / N_{0}$ is selected, its performance diverges from SCO-1 as the

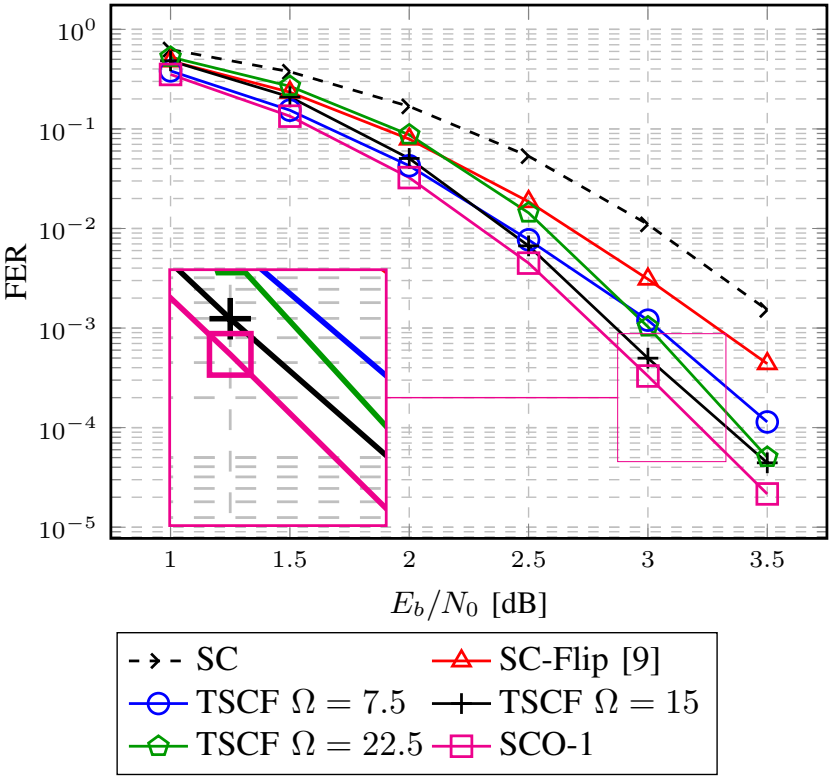

Fig. 16: FER curves with various decoding algorithms for $P C(1024,170) . C=7$ and $T_{\max }=10$ for all SC-Flip based implementations.

$E_{b} / N_{0}$ decreases. Finally, a moderate $\Omega$ value can maintain an acceptable FER for both low and high $E_{b} / N_{0}$ points; in case of Fig. 16, $\Omega=15$ yields an error-correction performance that is close to the SCO-1 performance at all considered $E_{b} / N_{0}$ values.

Fig. 17 depicts the error-correction performance of TSCF decoding against SC, SC-Flip, SC-Flip-EIS [12] and SC-List $(L=2)$ decoding for $P C(1024,512)$. The CRC remainder length is chosen to be $C=8$ for SC-List decoder using [17], while $C=12$ and $T_{\max }=10$ for all SC-Flip based decoders. Compared to Fig. 15 where the performance comparison for low-rate codes is demonstrated, the performance gap between SC-Flip and SCO-1 FER curves is smaller. This is due to the fact that the number of $E_{1}$ errors decreases as the rate increases, as highlighted earlier in Fig. 4. It can be seen that TSCF has superior error-correction performance compared to SC-Flip and SC-Flip-EIS, as it is closest to SCO1 performance. Moreover, the curve obtained SC-List with $L=2$ matches that of TSCF. Finally, SC-Flip-EIS decoding has a worse error-correction performance than SC-Flip: the effectiveness of the criteria described in [12] degrades as the code rate increases, since the size of critical set increases with rate and SC-Flip-EIS does not use any further restrictions to reduce the search space for the flipping index.

Fig. 18 presents the $E_{b} / N_{0}$ requirements to achieve target $\mathrm{FER}_{t}=10^{-4}$ for $N=1024, R \in\left\{\frac{1}{6}, \frac{1}{4}, \frac{1}{3}, \frac{1}{2}, \frac{2}{3}\right\}$ under different decoding approaches. For TSCF decoding, a single $\Omega$ is selected for each rate from Table III, regardless of the $E_{b} / N_{0}$ value. According to Fig. 18, TSCF decoding performs very close to the SCO-1 in all cases. As mentioned in Section III-C, the FER of SC-Flip approaches to that of SCO-1 as the rate increases. On the other hand, the benefit of TSCF decoding in terms of error-correction performance is maximum at $R=\frac{1}{4}$, with an improvement of $0.45 \mathrm{~dB}$ 


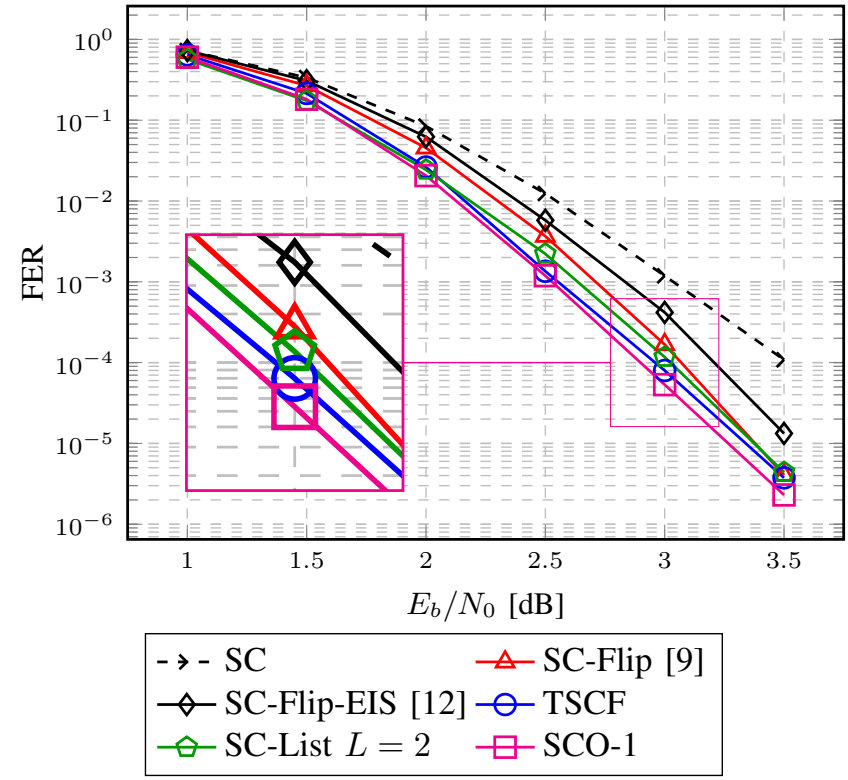

Fig. 17: FER curves with various decoding algorithms for $P C(1024,512) . C=12$ for all SC-Flip based decoders including SCO-1, $C=8$ for SC-List decoder, and $T_{\max }=10$ for SC-Flip, SC-Flip-EIS and TSCF decoding.

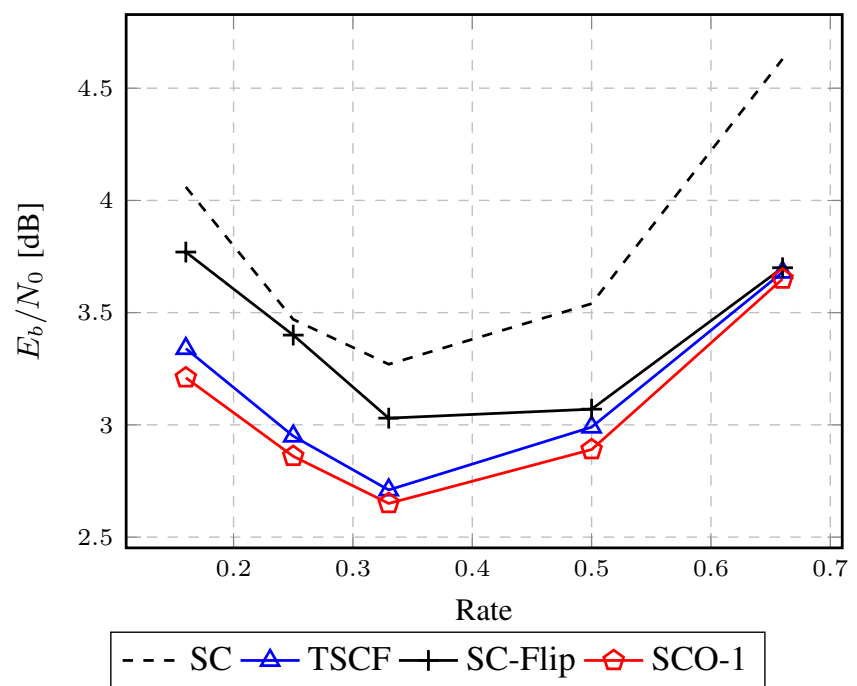

Fig. 18: $E_{b} / N_{0}$ requirements for different decoding approaches to achieve $\mathrm{FER}_{t}=10^{-4}$, for $N=1024, \Omega \in$ $\{15,15,12.5,10,10\}$ and $T_{\max }=10$ for $R \in\left\{\frac{1}{6}, \frac{1}{4}, \frac{1}{3}, \frac{1}{2}, \frac{2}{3}\right\}$, respectively.

compared to baseline SC-Flip decoding. Finally, compared to SC decoding, TSCF decoding has the highest gain at $R=\frac{2}{3}$ $(0.95 \mathrm{~dB})$.

\section{B. Complexity}

The (average) computational complexity of SC-Flip is directly proportional to the (average) number of iterations. A single iteration of SC-Flip consists of SC and CRC decoding, and an additional selection and sorting process for the flipping indices based on their LLR value when the initial CRC

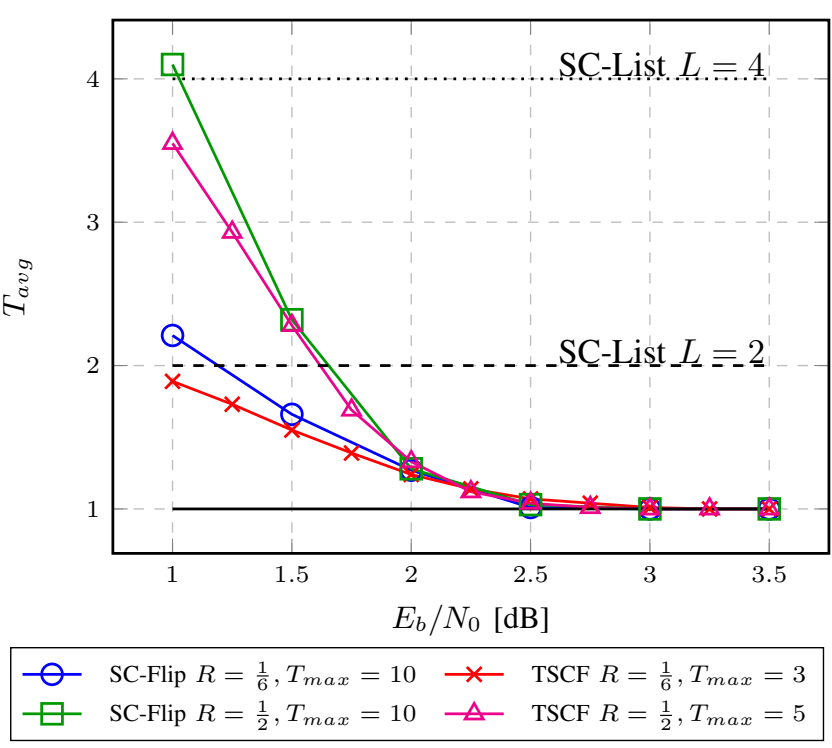

Fig. 19: $T_{\text {avg }}$ for SC-Flip and TSCF, $R=\left\{\frac{1}{6}, \frac{1}{2}\right\}$, at matched FER.

decoding fails. On the other hand, an iteration of TSCF is composed of SC+CRC decoding, and a comparison for critical indices to identify whether the associated LLR is lower than $\Omega$. In this Section, we compare $T_{\text {avg }}$ for both algorithms at matching $T_{\max }$ and at matching FER.

Fig. 19 presents the average number of iterations for both SC-Flip and TSCF decoding, for $R=\frac{1}{6}$ and $R=\frac{1}{2}$ at matching FER $=10^{-4}$. $T_{\max }$ of SC-Flip is kept at 10 and $T_{\max }$ of TSCF is tuned to match the error-correction performance of SC-Flip. For $R=\frac{1}{6}\left(R=\frac{1}{2}\right)$, the FER of TSCF matches that of SC-Flip when $T_{\max }=3\left(T_{\max }=5\right)$. As $E_{b} / N_{0}$ is increased, the average number of iterations converges to 1 , regardless of rate and decoding algorithm. In all cases, TSCF converges to $T_{\text {avg }}=1$ quicker than SC-Flip algorithm, with up to $14 \%$ lower $T_{\text {avg }}$ for $R=\frac{1}{6}$ and $R=\frac{1}{2}$.

Fig. 20 compares the time complexity of SC-Flip and TSCF decoders with SC, SC-List and Adaptive SC-List decoders, for $P C(1024,512)$. According to [1], $2 N-2$ time steps are needed to complete a full SC tree traversal. Consequently, both TSCF and SC-Flip implementations converge to 2046 average time steps with increasing $E_{b} / N_{0}$. On the other hand, $2 N+$ $K-2$ time steps are needed to complete one iteration of SCList decoding [4]. Thus, although SC-Flip and TSCF take more time steps on average at low $E_{b} / N_{0}$ values, they consume less time after $E_{b} / N_{0}=2 \mathrm{~dB}$. Compared to Adaptive SCList [18], TSCF has up to $83 \%$ more time steps; however the gap is closed quickly with growing $E_{b} / N_{0}$. At moderate-tohigh $E_{b} / N_{0}$, TSCF is shown to have $25 \%$ less time steps than Adaptive SC-List. Finally, at matched FER, TSCF requires up to $14 \%$ less time steps than SC-Flip implementation on average.

Table $\mathrm{V}$ reports $T_{\max }$ and the maximum $T_{\text {avg }}$ observed among the considered $E_{b} / N_{0}$ points, denoted by $T_{a v g}^{\max }$. The $\Omega$ values are selected from Table III, according to Fig. 18 . The FER of both algorithms matches in all cases. According to the results, at FER $=10^{-4}$ TSCF has both lower $T_{\max }$ (up 


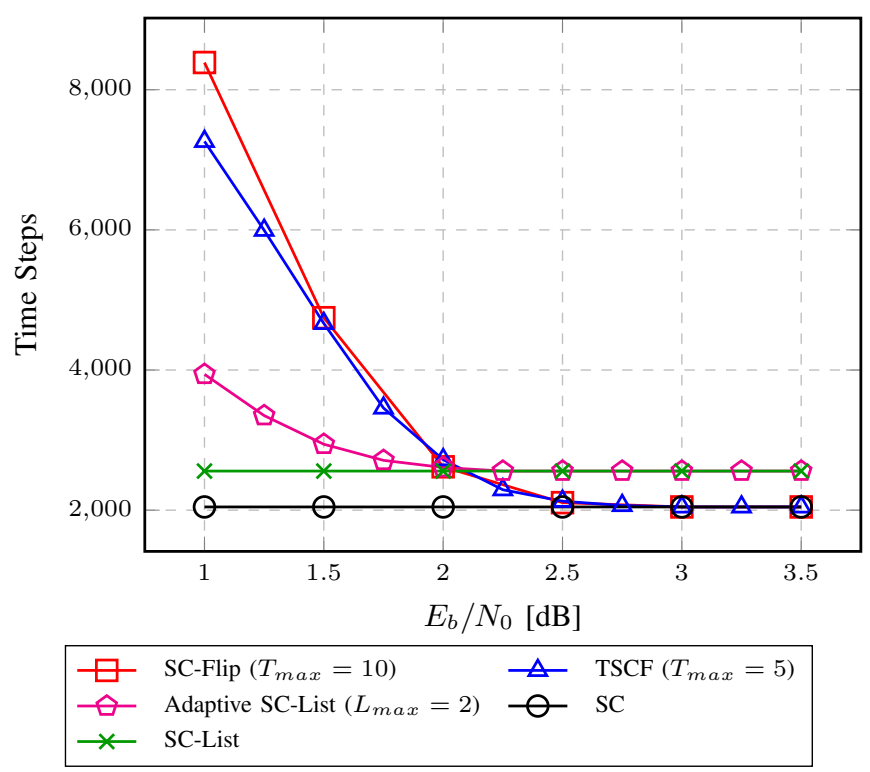

Fig. 20: Time complexity comparison of SC-Flip and TSCF for $P C(1024,512)$ with SC, SC-List and Adaptive SC-List decoders.

TABLE IV: $T_{a v g}^{\max }$ values for TSCF and SC-Flip at matching $T_{\max }=10$.

\begin{tabular}{lllllll}
\hline Rate $(R)$ & & $1 / 6$ & $1 / 4$ & $1 / 3$ & $1 / 2$ & $2 / 3$ \\
\cline { 1 - 4 } SC-Flip & & 2.21 & 2.42 & 2.59 & 4.10 & 6.55 \\
TSCF & & 3.02 & 2.95 & 3.04 & 5.18 & 8.20 \\
\hline
\end{tabular}

TABLE V: Observed $T_{\max }$ and worst case average number of iterations $\left(T_{\text {avg }}^{\max }\right)$ values for TSCF at matched error-correction performance with SC-Flip decoding at FER $=10^{-4}$.

\begin{tabular}{clcccccc}
\hline & Rate & & $1 / 6$ & $1 / 4$ & $1 / 3$ & $1 / 2$ & $2 / 3$ \\
\cline { 1 - 4 } \cline { 8 - 9 } SC-Flip & $T_{\max }$ & & 10 & 10 & 10 & 10 & 10 \\
& $T_{\text {avg }}^{\max }$ & & 2.21 & 2.42 & 2.59 & 4.10 & 6.55 \\
\hline \multirow{3}{*}{ TSCF } & $\Omega$ & & 15 & 12.5 & 15 & 15 & 12.5 \\
& $T_{\max }$ & 3 & 2 & 3 & 5 & 7 \\
& $T_{\text {avg }}^{\max }$ & & 1.80 & 1.45 & 1.97 & 3.53 & 6.13 \\
\hline
\end{tabular}

to $5 \times$ ) and $T_{a v g}$ (up to $40 \%$ ) at the same time. For matching $T_{\max }$, while TSCF outperforms SC-Flip in terms of FER, it has higher $T_{\text {avg }}$, as reported in Table IV. For example, $T_{\text {avg }}$ for TSCF is $26 \%$ higher than that of SC-Flip at $E_{b} / N_{0}=1$ $\mathrm{dB}$ when $T_{\max }=10$ for $R=\frac{1}{2}$. This is due to the fact that TSCF tends to flip the indices located towards the left side of the polar code, where SC-Flip does not have such a constraint. Thus, each decoding attempt by TSCF will averagely redecode more bits and contribute more to the total $T_{\text {avg }}$.

Table VI presents the $T_{\max }$ values and $E_{b} / N_{0}$ gain for TSCF for when $T_{a v g}^{\max }$ of SC-Flip and TSCF match at all $E_{b} / N_{0}$ values. $T_{\max }$ for SC-Flip is kept constant at 10 . Under these constraints, depending on the code rate, TSCF has up to $2.5 \times$ lower worst case latency than SC-Flip, with a concurrent $E_{b} / N_{0}$ gain up to $0.39 \mathrm{~dB}$.

TSCF shows improved error-correction performance and
TABLE VI: $T_{\max }$ values and $E_{b} / N_{0}$ gain at FER $=10^{-4}$ for TSCF compared to SC-Flip at matching $T_{a v g}^{\max }$ and SC-Flip $T_{\max }=10$.

\begin{tabular}{lcccccc}
\hline Rate $(R)$ & & $1 / 6$ & $1 / 4$ & $1 / 3$ & $1 / 2$ & $2 / 3$ \\
\cline { 1 - 4 }$T_{\max }$ & 4 & 4 & 5 & 6 & 7 \\
$E_{b} / N_{0}$ Gain $(\mathrm{dB})$ & 0.37 & 0.39 & 0.28 & 0.05 & 0.01 \\
\hline
\end{tabular}

reduced $T_{\max }$ requirements when compared to the SCF-EIS and SCF-FIS criteria in [12]. Compared to the technique in [13] targeting SCO-1, our methodology approaches the SCO-1 performance with significantly lower number of $T_{\max }$. Similarly, when compared to [11] targeting SCO-1, TSCF decoding yields similar error-correction performance at lower $T_{\max }$. Finally, TSCF has comparable error-correction performance to the PSCF algorithm from [14], with lower $T_{\max }$ requirements.

\section{CONClusion}

In this work, we have presented a thresholded index selection criterion that can be applied to SC-Flip decoding of polar codes, creating the thresholded SC-Flip (TSCF) algorithm. It is based on the identification of a set of error-prone bit indices and of an LLR threshold to determine the position of a channel-induced wrong bit estimation. We show how to optimize the threshold value, and how to efficiently select the erroneous indices. It is estimated to reduce the implementation complexity of SC-Flip. Simulations are performed over a wide range of rates, and demonstrate negligible performance loss for TSCF decoding compared to SCO-1 performance, with less than 10 maximum number of iterations. At matching maximum number of iterations, TSCF decoding has up to $0.45 \mathrm{~dB}$ gain in FER, compared to SC-Flip. At matching $\mathrm{FER}=10^{-4}$, TSCF requires up to $5 \times$ lower maximum number of iterations and has up to $40 \%$ lower average iterations. At matching $T_{\text {avg }}^{\max }$, TSCF requires up to $60 \%$ lower maximum number of iterations, with an $E_{b} / N_{0}$ gain of up to $0.39 \mathrm{~dB}$. Given the reduced computational complexity, reduced number of iterations and improved error-correction performance, TSCF is a good polar code decoding algorithm candidate for upcoming $5 \mathrm{G}$ applications.

\section{ACKNOWLEDGEMENTS}

The authors would like to thank Seyyed Ali Hashemi and Harsh Aurora for their valuable contributions to this work.

\section{REFERENCES}

[1] E. Arikan, "Channel polarization: A method for constructing capacityachieving codes for symmetric binary-input memoryless channels," IEEE Transactions on Information Theory, vol. 55, no. 7, pp. 3051-3073, July 2009.

[2] 3GPP, "Final report of 3GPP TSG RAN WG1 \#87 v1.0.0," http://www.3gpp.org/ftp/tsg_ran/WG1_RL1/TSGR1_87/Report/Final_ Minutes_report_RAN1 $\backslash \% 2387 \_v 100 . z i p$, Reno, USA, November 2016.

[3] I. Tal and A. Vardy, "List decoding of polar codes," IEEE Transactions on Information Theory, vol. 61, no. 5, pp. 2213-2226, May 2015.

[4] A. Balatsoukas-Stimming, M. B. Parizi, and A. Burg, "LLR-based successive cancellation list decoding of polar codes," IEEE Transactions on Signal Processing, vol. 63, no. 19, pp. 5165-5179, Oct 2015. 
[5] S. A. Hashemi, C. Condo, and W. J. Gross, "Fast simplified successivecancellation list decoding of polar codes," in 2017 IEEE Wireless Communications and Networking Conference Workshops (WCNCW), pp. 1-6, March 2017.

[6] S. A. Hashemi, C. Condo, and W. J. Gross, "Fast and flexible successivecancellation list decoders for polar codes," IEEE Transactions on Signal Processing, vol. 65, no. 21, pp. 5756-5769, Nov 2017.

[7] S. A. Hashemi, M. Mondelli, S. H. Hassani, R. Urbanke, and W. J. Gross, "Partitioned list decoding of polar codes: Analysis and improvement of finite length performance," pp. 1-7, Dec 2017.

[8] F. Ercan, C. Condo, S. A. Hashemi, W. J. Gross, "On error-correction performance and implementation of polar code list decoders for 5G," in 55th Annual Allerton Conference on Communication, Control, and Computing (Allerton), pp. 443-449, Oct 2017.

[9] O. Afisiadis, A. Balatsoukas-Stimming, and A. Burg, "A low-complexity improved successive cancellation decoder for polar codes," in Asilomar Conference on Signals, Systems and Computers, pp. 2116-2120, Nov 2014.

[10] L. Chandesris, V. Savin, and D. Declercq, "An improved SCFlip decoder for polar codes," in IEEE Global Communications Conference (GLOBECOM), pp. 1-6, Dec 2016.

[11] L. Chandesris, V. Savin, and D. Declercq, "Dynamic-SCFlip decoding of polar codes," IEEE Transactions on Communications, vol. 66, no. 6, pp. 2333-2345, June 2018.

[12] C. Condo, F. Ercan, and W. J. Gross, "Improved Successive Cancellation Flip Decoding of Polar Codes Based on Error Distribution,"2018 IEEE Wireless Communications and Networking Conference Workshops (WCNCW), pp. 19-24, Apr 2018.

[13] Z. Zhang, K. Qin, L. Zhang, H. Zhang, and G. T. Chen, "Progressive bit-flipping decoding of polar codes over layered critical sets," in GLOBECOM 2017 - 2017 IEEE Global Communications Conference, pp. 1-6, Dec 2017.

[14] F. Ercan, C. Condo, S. A. Hashemi, and W. J. Gross, "Partitioned Successive-Cancellation Flip Decoding of Polar Codes," in IEEE International Conference on Communications (ICC), pp. 1-6, May 2018.

[15] I. Tal and A. Vardy, "How to construct polar codes," IEEE Transactions on Information Theory, vol. 59, no. 10, pp. 6562-6582, Oct 2013.

[16] P. Giard, A. Balatsoukas-Stimming, T. C. Mller, A. Bonetti, C. Thibeault, W. J. Gross, P. Flatresse, and A. Burg, "Polarbear: A $28 \mathrm{~nm}$ fd-soi asic for decoding of polar codes," IEEE Journal on Emerging and Selected Topics in Circuits and Systems, vol. 7, no. 4, pp. 616-629, Dec 2017.

[17] S. A. Hashemi, C. Condo, F. Ercan, and W. Gross, "On the performance of polar codes for 5G eMBB control channel," in Asilomar Conference on Signals, Systems and Computers, pp. 1764-1768, 2017.

[18] B. Li, H. Shen and D. Tse, "An adaptive successive cancellation list decoder for polar codes with cyclic redundancy check," IEEE Communications Letters, vol. 16, no. 12, pp. 2044-2047, Dec 2012.

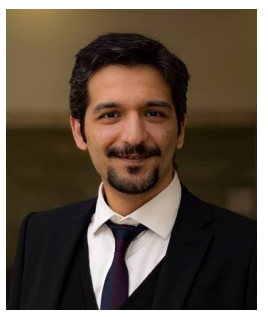

Furkan Ercan (S'11) received the B.Sc. degree in Electrical and Electronics Engineering in 2011 and M.Sc. degree in Sustainable Environment and Energy Systems in 2015, both from Middle East Technical University (METU) Northern Cyprus Campus (NCC), Ankara, Turkey. From 2011 to 2012, he worked as a full time R\&D intern at Intel Corporation in Hillsboro, OR, USA, focusing on systemlevel energy efficiency on enterprise platforms. He is currently pursuing a Ph.D. degree with McGill University, Montréal, QC, Canada. His research interests are algorithm, design and implementation of signal processing systems with a focus on polar codes, and energy aware hardware architectures. He received a Best Student Paper Award in 2015 IEEE International Conference in Energy Aware Computing (ICEAC) in Cairo, Egypt.

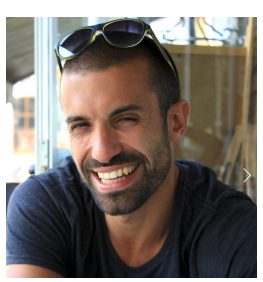

Carlo Condo (M'15) received the M.Sc. degree in electrical and computer engineering from the Politecnico di Torino and The University of Illinois at Chicago, Chicago, IL, USA in 2010, and the $\mathrm{Ph} . \mathrm{D}$. degree in electronics and telecommunications engineering from the Politecnico di Torino and IMT Atlantique in 2014. From 2015 to 2017, he was a Post-Doctoral Fellow with the ISIP Laboratory, McGill University, where he was a McGill University Delegate at the 3GPP meetings for the fifthgeneration wireless systems standard (5G) in 2017.

Since 2018, he has been a Researcher with the Communication Algorithms Design Team, Huawei Paris Research Center. His Ph.D. dissertation received a mention of merit as one of the five best of 2013/2014 by the GE association. He was a recipient of two conference best paper awards (SPACOMM 2013 and ISCAS 2016). His research is focused on channel coding, design and implementation of encoder and decoder architectures, digital signal processing, and machine learning.

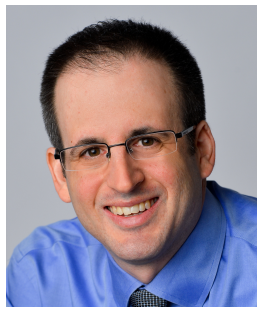

Warren J. Gross (S'92-M'04-SM'10) received the B.A.Sc. degree in electrical engineering from the University of Waterloo, Waterloo, ON, Canada, in 1996, and the M.A.Sc. and Ph.D. degrees from the University of Toronto, Toronto, ON, Canada, in 1999 and 2003, respectively. He is currently a Professor and the Chair of the Department of Electrical and Computer Engineering, McGill University, Montreal, QC, Canada. His research interests are in the design and implementation of signal processing systems and custom computer architectures.

Dr. Gross served as the Chair for the IEEE Signal Processing Society Technical Committee on Design and Implementation of Signal Processing Systems. He served as the General Co-Chair for the IEEE GlobalSIP 2017 and the IEEE SiPS 2017 and the Technical Program Co-Chair for SiPS 2012. $\mathrm{He}$ also served as an Organizer for the Workshop on Polar Coding in Wireless Communications at WCNC 2017, the Symposium on Data Flow Algorithms and Architecture for Signal Processing Systems (GlobalSIP 2014), and the IEEE ICC 2012 Workshop on Emerging Data Storage Technologies. He served as an Associate Editor for the IEEE TRANSACTIONS ON SIGNAL PROCESSING and as a Senior Area Editor. He is a Licensed Professional Engineer in the Province of Ontario. 